Naturwissenschaften 76, 172-173(1989) ๑ Springer-Verlag 1989

\section{ESR Study of Fe(III) and Cr(III) Hydroxides}

\author{
A. U. Gehring \\ EAWAG/ETH, CH-8600 Dübendorf
}

\author{
R. Karthein \\ Laboratorium für Physikalische Chemie der ETH, CH-8092 Zürich
}

Electron spin resonance spectroscopy (ESR) has seldom been used to study hydroxide compounds. It has been shown that ESR can be applied to obtain information about the structure of ferric solids [1]. However, ESR data of $\mathrm{Fe}$ (III) and $\mathrm{Cr}$ (III) hydroxides have not been considered thus far. Many structural data of $\mathrm{Fe}$ (III) and $\mathrm{Cr}$ (III) hydroxides can be derived from X-ray and calorimetric experiments [2]. The existence of mixed phases was assumed based on solubility data of coprecipitated amorphous $\mathrm{Fe}$ (III)-Cr(III) hydroxides [3]. This note presents ESR data of $\mathrm{Fe}($ III) and $\mathrm{Cr}$ (III) hydroxides and a mixed phase for temperatures between room temperature and $150^{\circ} \mathrm{C}$.

The solid phases were prepared by rapid titration of $0.05 \mathrm{M}$ iron nitrate, chromium nitrate and a $1: 1$ mixture of the two solutions with $1 M \mathrm{NaOH}$ to a $\mathrm{pH}$ of about 10 . The precipitates were subsequently stored at room temperature for 20 days, then washed from electrolytes and dried at $40^{\circ} \mathrm{C}$. A $\mathrm{Fe}$ (III)/Cr(III) ratio of about 1 in the mixed phase was determined by atomic absorption spectrometry (AAS) after acid dissolution. The structural analysis of the solids was performed by X-ray diffractometry (XRD; Guinier-IV camera with $\mathrm{Cu} K_{\alpha}$ and/or $\mathrm{Fe} K_{\alpha}$ radiation) and by differential scanning calorimetry (DSC) prior to the ESR measurement.

$\alpha-\mathrm{FeOOH}$ (goethite) and $\mathrm{Cr}(\mathrm{OH})_{3}$ were the two crystallized pure phases identified by XRD, whereas the mixed phase is amorphous. Calorimetry in the temperature range between 0 and $200^{\circ} \mathrm{C}$ shows different thermal behavior for the three solids (Fig. 1). No significant enthalpy changes up to $200^{\circ} \mathrm{C}$ were detected with $\alpha$-FeOOH and the mixed phase. The broad peak between 0 and $80^{\circ} \mathrm{C}$ of the amorphous mixed phase can be explained by loss of sorbed moisture. DSC of $\mathrm{Cr}(\mathrm{OH})_{3}$ on the other hand reveals an endotherm peak at about $110^{\circ} \mathrm{C}$. This enthalpy change indicates a structural conversion of the $\mathrm{Cr}(\mathrm{OH})_{3}$. XRD reveals that the slightly broadened diffraction lines of $\mathrm{Cr}(\mathrm{OH})_{3}$ disappeared after the thermal treatment. This suggests the formation of a new $\mathrm{X}$-ray amorphous phase.

ESR spectra of the solids at room temperature consist of isotropic lines with $g$-values of about 2 (Fig. $2 \mathrm{~A}-\mathrm{C}, a$ ). $\alpha$ FeOOH shows a linewidth of 300 Gauss

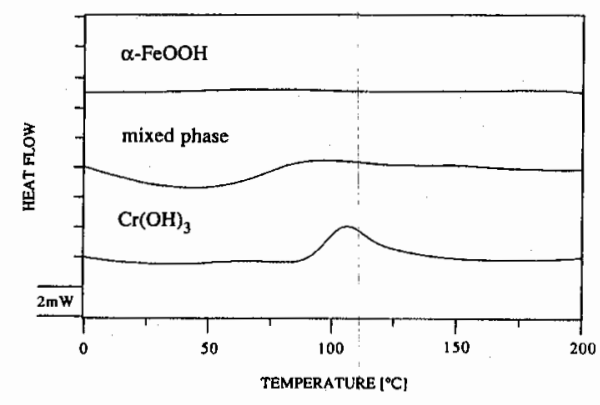

Fig. 1. Differential scanning calorimetry of the solid phases; 15 -mg sample, heating rate $10^{\circ} \mathrm{C} \mathrm{min}^{-1}$
(Fig. 2A). Upon heating within the spectrometer cavity, the ESR amplitude of the $\mathrm{Fe}$ (III) in the goethite shows a slight decrease as expected from Boltzmann statistics. During cooling to room temperature, the intensity reaches almost the initial value.

At room temperature, the ESR of $\mathrm{Cr}(\mathrm{OH})_{3}$ shows a relatively narrow signal with a linewidth of 150 Gauss (Fig. $2 \mathrm{~B}, a)$. No significant change of the signal is found up to $70^{\circ} \mathrm{C}$ (Fig. 2B, b). Above this temperature a spectral change is observed. At $150^{\circ} \mathrm{C}$ a new lineshape forms, with a drastic widening of the linewidth to 450 Gauss (Fig. 2B, c). This indicates a structural conversion of the ligand sphere of $\mathrm{Cr}^{3+}$. The shape of the spectra is irreversible when cooling down to room temperature (Fig. 2B, d). Therefore, the different signals of the room temperature measurements before and after thermal treatment (Fig. 2B, $a, d$ ) can be explained by the formation of $a$ new phase. These ESR results are in good agreement with DSC and XRD. Furthermore, it is shown that ESR can be used as an analytical tool to study structural conversions in $\mathrm{Cr}$ (III) hydroxide systems.

In contrast to the $\alpha-\mathrm{FeOOH}$ and $\mathrm{Cr}(\mathrm{OH})_{3}$, the mixed phase reveals a broadened ESR signal of 1330 Gauss at room temperature (Fig. 2C, a). Based on AAS, this phase can be described as $\mathrm{Fe}_{0.5} \mathrm{Cr}_{0.5}(\mathrm{OH})_{3}$. Upon heating the ESR signal shows only a decrease of the peak intensity similar to that found for $\alpha$-FeOOH. At high temperature, no change of the linewidth is observed, in contrast to $\mathrm{Cr}(\mathrm{OH})_{3}$. Furthermore, the signal of the mixed phase is restored when cooling down to room temperature. The ESR spectra of the mixed phase show different behavior during thermal treatment as compared to the pure $\mathrm{Cr}$ (III) hydroxide. Therefore, it can be concluded that the coprecipitated $\mathrm{Fe}(\mathrm{III})$ and $\mathrm{Cr}$ (III) form a single solid phase. 

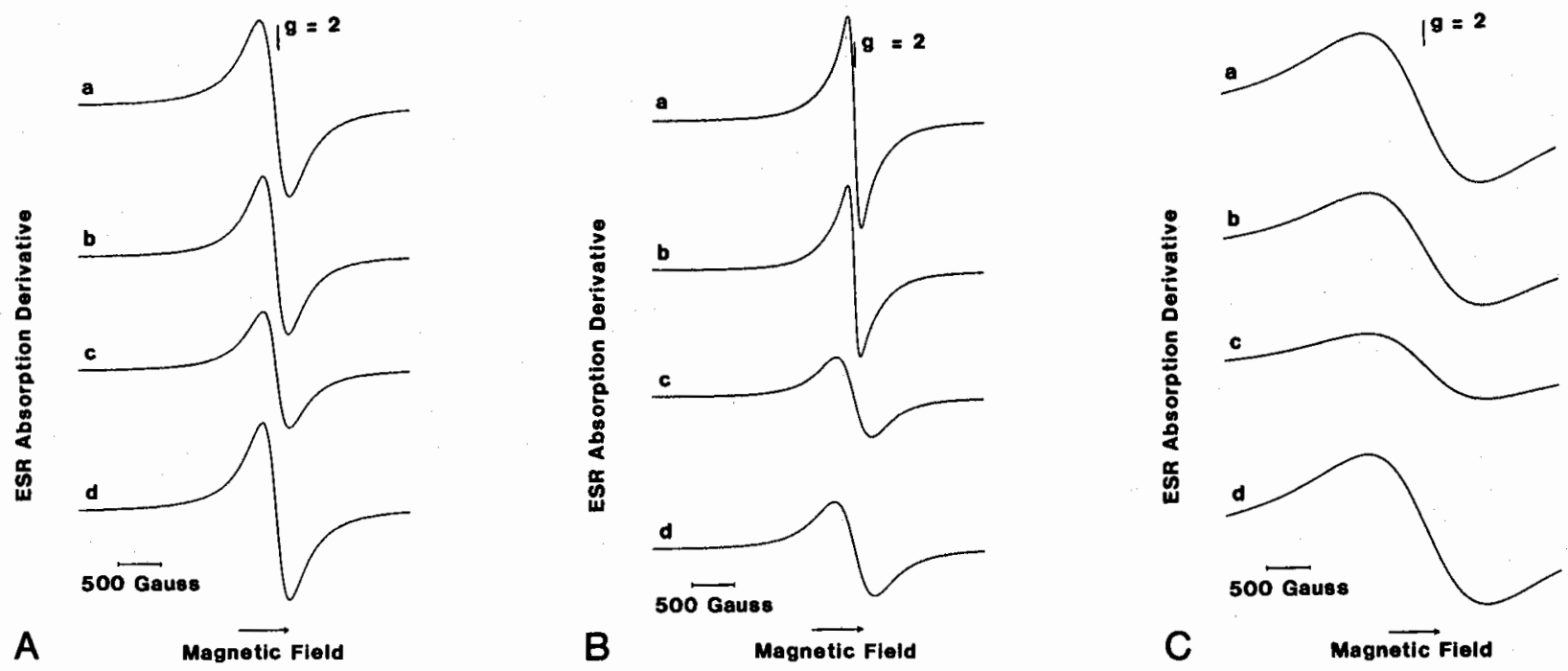

Fig. 2. ESR spectra of A) $\alpha-\mathrm{FeOOH}, \mathrm{B}) \mathrm{Cr}(\mathrm{OH})_{3}$, C) the mixed phase at room temperature $(a), 70^{\circ} \mathrm{C}(b), 150^{\circ} \mathrm{C}(c)$, and at room temperature after thermal treatment $(d)$

In summary, ESR provides strong evidence for the occurrence of a $\mathrm{Fe}(\mathrm{III})-\mathrm{Cr}$ (III) hydroxide, and clearly shows that this phase is different from the $\mathrm{Fe}(\mathrm{III})$ oxyhydroxide and the Cr(III) hydroxide.
The authors would like to thank Dr. A. Reller and B. Spring (University of Zürich) for providing the DSC analysis. This research was supported by the Swiss National Foundation, project no. 2.942-0.88 and by a grant of the Board of the Swiss Federal Institute of Technology.
Received January 23, 1989

1. Pinheiro, E. A., et al.: Langmuir 3, 445 (1987)

2. Alario Franco, M. A., Sing, K. S. W.: J. Therm. Anal. 4, 47 (1971)

3. Sass, B. M., Rai, D.: Inorg. Chem. 26, 2228 (1987) 